\title{
Intra-abdominal abscesses secondary to Streptococcus anginosus infection in a postpartum patient: A case report and review of the literature
}

\author{
Rehab Shabana ${ }^{1}$, Lindsay Berbiglia ${ }^{2}$, John Barnwell ${ }^{3}$, Ronald Cheek ${ }^{1}$, Mark Wolf ${ }^{4}$, \\ Bernard Gonik ${ }^{1}$ \\ ${ }^{1}$ Department of Obstetrics and Gynecology, Wayne State University School of Medicine/Detroit Medical Center, Detroit, USA \\ ${ }^{2}$ Department of General Surgery, Detroit Medical Center, Detroit, USA \\ ${ }^{3}$ Department of General Surgery, Wayne State University School of Medicine/Detroit Medical Center, Detroit, USA \\ ${ }^{4}$ Department of Internal Medicine, Detroit Medical Center, Detroit, USA \\ Email: rshabana@med.wayne.edu
}

Received 24 March 2012; revised 30 April 2012; accepted 11 May 2012

\begin{abstract}
Although rare, infection with Streptococcus anginosus has a known predilection for abscess formation. We report here a case of a 20 year-old, otherwise healthy female patient noted to have developed abdominal and pelvic abscesses secondary to Streptococcus anginosus infection. Although the inciting focus of the pathogen remains unclear in our patient, this case report emphasizes the importance of rapid identification of the organism and highlights the approach to therapeutic options in the management of such cases.
\end{abstract}

Keywords: Streptococcus anginosus; Abdominal Abscess

\section{CASE}

A 20 year-old G4 P3-0-1-3 presented to the emergency department seven days after spontaneous vaginal delivery of a term infant, complaining of worsening lower abdominal pain since hospital discharge. Aside from a first degree perineal laceration, which was repaired uneventfully, the entire prenatal course and delivery were otherwise uncomplicated. She denied fever, nausea, vomiting or change in bowel function. Vital signs were stable except for a resting tachycardia of $118 \mathrm{bpm}$. Temperature was $36.3^{\circ} \mathrm{C}$. Pertinent physical exam findings included a distended abdomen which was soft, mildly tender in all quadrants, with bowel sounds present, and without rebound or guarding. A small non-tender, reducible umbilical hernia was also appreciated on exam. The uterus was well involuted below the umbilicus and non-tender. Pelvic exam revealed small, non-foul smelling lochia and no cervical motion or adnexal tenderness.
There was bulging noted in the posterior vaginal fornix. The perineal repair was healing well, without induration or erythema. Laboratory studies included a white blood cell count of 21,000 cells per liter and a hemoglobin of 10.5 grams. The urinalysis was unremarkable, and subsequent urine and blood cultures were negative. She was HIV negative by antibody testing.

The patient underwent ultrasonography which revealed a normal appearing postpartum uterus and adnexa. A large amount of free fluid was seen in the abdomen and pelvis. The appendix was not visualized. The CT scan demonstrated multiple areas of loculated fluid pockets (Figure 1). Again, the appendix was unable to be visualized. The patient was empirically started on cefoxitin in the emergency department. She was then continued on metronidazole and ampicillin therapy upon

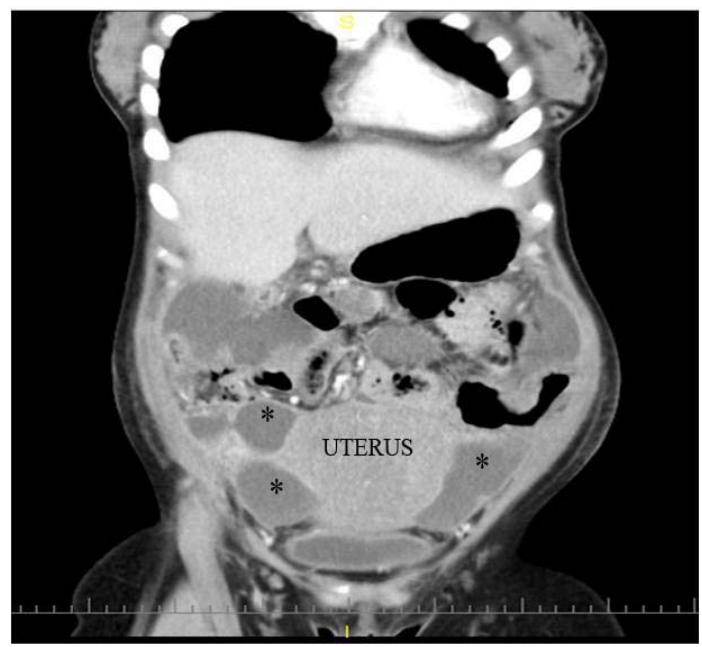

Figure 1. Admission computed tomography scan demonstrating multiple fluid collections in the peritoneal cavity as denoted by asterisks. 
admission, and switched to Ampicillin-Sulbactam on the fifth day of admission. A culdocentesis was performed with drainage of $420 \mathrm{cc}$ of purulent, foul-smelling fluid. Cultures yielded Streptococcus anginosus and Prevotella species. After surgery and interventional radiology consultations, three indwelling 10-French pigtail catheters were placed transabdominally at sites thought to contain the largest fluid collections by CT imaging. Over 200 cc of purulent fluid were initially collected at each of these drainage sites, with steadily diminishing amounts subsequently. Aerobic and anaerobic cultures were negative from all of these aspirates. At the drainage sites, abscess resolution was noted later in the hospital course, while other collections were re-demonstrated on CT imaging (Figure 2). Throughout this time, the patient remained stable, with improving symptomatology. Occasional low grade temperature elevations were noted, without a newly identified source. On hospital day 15, the previously noted umbilical hernia became tender to palpation with spontaneous drainage of purulent material similar to that noted above. An associated fluid collection measuring $2.6 \mathrm{~cm} \times 3.4 \mathrm{~cm}$ was identified. Cultures were again negative. The patient was taken to the operating room fordrainage of this collection and umbilical hernia repair. The collection was drained, copiously irrigated with normal saline solution, and the fascial umbilical defect was repaired with the skin left open. On hospital day 20, the patient continued to be afebrile, was tolerating diet and showed resolution of her abdominal pain. She was discharged home on broad spectrum oral antibiotic therapy with drains remaining in place. One week later, at her

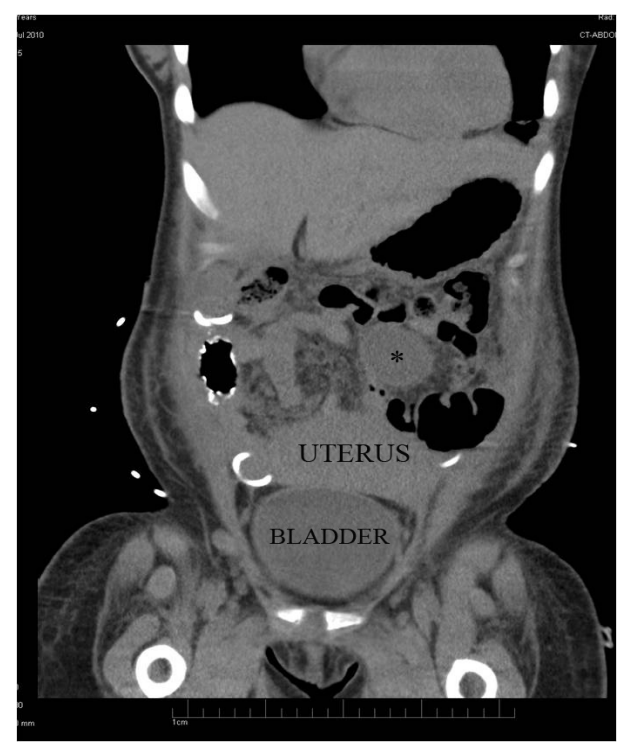

Figure 2. Hospital day 13 computed tomography scan after placement of drainage catheters. Resolution of right and left lower quadrant collections visualized. A discrete fluid collection within the mesenteric space is noted $\left(^{*}\right)$. scheduled follow up office visit, the patient had no complaints and drains were removed.

\section{DISCUSSION}

Streptococcus anginosus group, also known as S. milleri, are a gram positive cocci with known commensal colonization of the mouth, nasopharynx, gastrointestinal tract, and lower genital tract, with isolation rates ranging from $15 \%-30 \%[1,2]$. This group consists of three species: $S$. anginosus, S. constellatus, and S. intermedius, and several subspecies [3]. Though all members of the Streptococcus anginosus group share common phenotypic characteristics, confusion surrounding serologic and hemolytic characterization persists. Currently proposed methods for identification of the Streptococcus anginosus group, to the species level in particular, are time consuming and not routinely done in clinical practice. This has lead to incomplete or misidentification of this group, often as $S$. viridans organisms, which have different predilection and resistance patterns [4]. This is of significant concern, in that the lack of recognition can lead to mismanagement of patients with life-threatening conditions.

The unique characteristic of the $S$. anginosus group, distinguishing it from other streptococci, is its role in pyogenic infections and its strong association with abscess formation. S. anginosus produces deep-seated abscesses in a frequency double that of the remainder of the S. viridians group [5]. Serious underlying disease, such as immunodeficiency, malignancy, and diabetes, as well as, recent surgical intervention are predisposing factors in $33 \%-76 \%$ of S. anginosus infections [1]. Trauma to the mucosal barrier and intra-abdominal infections, including appendicitis and cholecystitis, were noted inciting foci, although abscess formation may occur without an obvious source [5-7].

Few reports of $S$. anginosus group infections are identified in a healthy obstetric and gynecologic patient population. Isolated cases of wound infection, pelvic abscesses, labial abscesses, and tuboovarian abscesses have been previously described $[1,8]$. Of interest, three cases of epidural abscesses have been reported in obstetric patients, of which one was at 20 weeks of gestation, occurring as a spontaneous event without catheterization or other invasive procedures. This case is of particular interest due to the patient's lack of predisposing conditions and quick clinical deterioration [9]. The other two cases involved young healthy women after spontaneous vaginal deliveries with epidural catheter placement for analgesia. Both underwent laminectomy and treatment with antibiotics [8]. There have also been limited case reports of neonatal sepsis involving this organism with the presumption of vertical transmission from a colonized mother [10]. S. anginosus may also play a role in causing septic 
abortions via ascending infection [11].

The case presented is of particular interest for several reasons including the lack of obvious inciting cause, the disparity between the patient's eventually recognized disease state and her presenting symptomatology, and the choice of management. Treatment with systemic antibiotics and serial interventional radiology-directed drainage versus early surgical exploration remains controversial. It has been noted in previous studies, that the majority of infectious $S$. anginosus cases originate from skin/soft tissue or intraabdominal sources. Of 186 cases of $S$. anginosus noted in a retrospective study, 64 had skin/soft tissue manifestations and 41 had intraabdominal manifestations. These included liver abscess, cholangitis, peritonitis, pancreatic abscess, subphrenic abscess, and most commonly appendicitis, the source in 23 of the 41 cases [1]. Another study detailing 33 cases of S. anginosus and bacteremia found the majority were due to intraabdominal sources [7]. The inciting factors in these cases included cholecystitis/cholangitis (18\%) and appendicitis (12\%) [7]. Given our patient's extensive intraabdominal disease process, appendicitis was considered a possible inciting focus. The patient underwent several imaging procedures, including ultrasonography and serial CT scanning, none of which allowed visualization of the appendix. Though not visualized, there was also no noted area of increased inflammation surrounding the area of the appendix to suggest appendicitis. Of note, $S$. anginosus is a commensal organism of the appendix and was isolated from a quarter of normal-appearing appendices, not in the setting of appendicitis [12]. Lastly, regarding a source of the infection, it should be recalled that the patient had a small perineal laceration repaired in an uncomplicated manner at the time of delivery. As previously noted, $S$. anginosus species are known to colonize the lower female genital tract. Therefore, trauma to this mucosal barrier may have been the site for entrance of the pathogen. Our patient did not have any signs of inflammation, induration or purulence at the laceration site upon subsequent presentation to the hospital. Of interest, certain S. anginosus biotypes have a specific affinity for the vaginal mucosa. They are identified by the fact they produce acid from raffinose and melibiose in the laboratory. Unfortunately our clinical specimen was not tested with these substrates to help better define the source of the organism.

A final point of interest in this case is the management of multiple intra-abdominal abscesses with percutaneous drainage instead of surgical intervention. On presentation the patient had significant abdominal tenderness, but lacked other gastrointestinal symptoms. She was tolerating a regular diet and complained of only mildly decreased appetite. She was afebrile and hemodynamically stable. Also, at the time of presentation, the patient had been symptomatic for seven days, making surgical intervention a potentially more complicated approach. Given the noted clinical findings and the apparent amenability of the fluid collections to drainage, the decision was made to proceed with percutaneous drainage.

On review, there are several studies detailing the use of radiology guided percutaneous drainage catheters for intraabdominal abscesses and success rates comparable to that of open intervention, with overall success rates between $33 \%$ and $100 \%$ [13]. Three studies were reviewed detailing the management of intraabdominal abscesses, leading to similar conclusions that percutaneous drainage had a higher success rate determined by abscess and sepsis resolution, and lower rates of complications, when compared to operative intervention [14-16]. It should be noted that second attempts at percutaneous drainage were required in a significant number of patients described in the above studies and that the duration of drain management ranged from 6 to 60 days. Our patient underwent a total of five drainage procedures and three drainage catheter placement procedures, which resulted in resolution of the fluid collections. Common adverse events described with catheter management included drains falling out or becoming clogged or damaged, cellulitis at the drain site, and less commonly fistula formation, bleeding, need for re-hospitalization, and inability to place the drain.

Recognized limitations in the evaluation of this case include the initiation of antibiotics prior to obtaining cultures and the polymicrobial nature of the infection. Intravenous antibiotic therapy was initiated prior to the culdocentesis procedure from which the S. anginosus cultures were obtained. This may have limited culture results, suppressing growth of other involved pathogens. Prevotella species was also isolated from the culdocentesis specimen. Its ability to cause abscesses and bacteremia is well documented, particularly in the mouth following invasive procedures. Since both $S$. anginosus and Prevotella both can colonize the oral cavity, a more careful assessment of the patient's teeth and mouth would have been helpful despite there being no presenting symptomatology related to these structures. With regard to antibiotic selection, the initial empiric use of broad spectrum antibiotics is justifiable given the presenting circumstances. Later, culture-based identification of the causative pathogens would have allowed for therapeutic modifications in the event of a suboptimal response, or if microbial resistance was identified. Fortunately, S. anginosus is sensitive to a wide variety of Gram-positive focused antimicrobial agents.

In summary, this case report details an unusual virulent infection presenting in an otherwise young healthy postpartum patient caused by $S$. anginosus. Because of the pathologic potential for this organism, it highlights 
the importance of careful laboratory identification and timely reporting. Given its predilection for purulence and abscess formation, once identified as a cause for infection, careful observation for disease progression is required. Transabdominal percutaneous drainage of accessible abscess pockets, in the otherwise stable parturient, seems to be a reasonable consideration as a first line approach. There are presently insufficient data available in the obstetric literature detailing colonization rates and the incidence of disease. This limits insight as to the need for preemptive identification or prophylactic interventions, as we do with other known puerperal Streptococcus pathogens $S$. agalactiae and S. pyogenes.

\section{REFERENCES}

[1] Singh, K.P., Morris, A., Lang, S.D., MacCulloch, D.M. and Bremner, D.A. (1988) Clinically significant Streptococcus anginosus (Streptococcus milleri) infections: A review of 186 cases. New Zealand Medical Journal, 101, 813-816.

[2] Ruoff, K.L. (1988) Streptococcus anginosus (Streptococcus milleri): The unrecognized pathogen. Clinical Microbiology Reviews, 1, 102-128.

[3] Claridge, J.E., et al. (2001) Streptococcus intermedius, Streptococcus constellatus, and Streptococcus anginosus (Streptococcus milleri group) are of different clinical importance and are not equally associated with abscess. Clinical Infectious Diseases, 32, 1511-1515. doi:10.1086/320163

[4] Facklam, R. (2002) What happened to the streptococci: Overview of taxonomic and nomenclature changes. Clinical Microbiology Reviews, 15, 613-630. doi:10.1128/CMR.15.4.613-630.2002

[5] Admon, D., Ephros, M.A., Gavish, D. and Raz, R. (1987) Infection with Streptococcus milleri. Journal of Infection, 14, 55-60. doi:10.1016/S0163-4453(87)90834-6

[6] Jacobs, J.A., Pietersen, H.G., Stobberingh, E.E. and Soeters, P.B. (1994) Bacteremia involving the "Streptococcus milleri” group: Analysis of 19 cases. Clinical Infectious Diseases, 19, 704-713. doi:10.1093/clinids/19.4.704
[7] Salavert, M., Gomez, L., Rodriguez-Carballeira, M., Xercavins, M., Freixas, N. and Garau, J. (1996) Seven-year review of bacteremia caused by Streptococcus milleri and other viridans streptococci. European Journal of Clinical Microbiology \& Infectious Diseases, 15, 365-371. doi:10.1007/BF01690091

[8] Treszezamsky, A.D. and Feldman, D., Sarabanchong, V.O. (2011) Concurrent postpartum uterine and abdominal wall dehiscence and Stretococcus anginosus infection. Obstetrics \& Gynecology, 118, 449-451. doi:10.1097/AOG.0b013e31821619e9

[9] Lampen, R. and Bearman, G. (2005) Epidural abscess caused by Streptococcus milleri in a pregnant woman. BMC Infectious Diseases, 5, 100. doi:10.1186/1471-2334-5-100

[10] Cox, R.A., Chen, K., Coykendall, A.L., Wesbecher, P. and Herson, V.C. (1987) Fatal infection in neonates of 26 weeks' gestation due to Streptococcus milleri: Report of two cases. Journal of Clinical Pathology, 40, 190-193. doi:10.1136/jcp.40.2.190

[11] MacGowan, A.P. and Terry, P.B. (1987) Streptococcus milleri and second trimester abortion. Journal of Clinical Pathology, 40, 292-293. doi:10.1136/jcp.40.3.292

[12] Poole, P.M. and Wilson, G. (1977) Streptococcus milleri in the appendix. Journal of Clinical Pathology, 30, 937942. doi:10.1136/jep.30.10.937

[13] Gohl, J., Gmeinwieser, J. and Gusinde, J. (1999) Intraabdominal abscesses. Intervention versus surgical treatment. Zentralblatt fur Chirurgie, 124, 187-194.

[14] Cinat, M.E., Wilson, S.E. and Din, A.M. (2002) Determinants for successful percutaneous image-guided drainage of intra-abdominal abscess. Archives of Surgery, 137, 845-849. doi:10.1001/archsurg.137.7.845

[15] Johnson, W.C., Gerzof, S.G., Robbins, A.H. and Nabseth, D.C. (1981) Treatment of abdominal abscesses: Comparative evaluation of operative drainage versus percutaneous catheter drainage guided by computed tomography or ultrasound. Annals of Surgery, 194, 510-520. doi:10.1097/00000658-198110000-00014

[16] Van Sonnenberg, E., et al. (1984) Percutaneous drainage of 250 abdominal abscesses and fluid collections. Part I: Results, failures, and complications. Radiology, 151, 337341. 